\title{
Comment survivre sans la figure paternelle ?
}

\section{Magali BLANC, Université de la Colombie Britannique}

Quel genre d'individu devenons-nous sans la présence de notre père ? Ancré dans la génétique de l'Homme, le père, figure représentative de l'autorité et de la loi, entretient une relation complexe avec ses enfants. Ce dernier n'ayant pas partagé de lien physique (le cordon ombilical) avec son enfant, il se voit incarner un rôle prédéterminé par rapport à celui de la mère. En d'autres termes, c'est parce que la mère représente de manière générale la douceur, l'amour et la fusion que le père est investi par des rôles plus durs. Tandem inséparable, le père et la mère forment un couple indispensable au bon développement de l'enfant, en un individu responsable, droit, juste et honnête. L'éducation reçue par ses parents devrait lui permettre d'être à son tour un adulte épanoui et prêt à affronter les défis de la vie. Cependant, il semblerait que pour les personnages d'Agaguk d'Yves Thériault et de La petite fille qui aimait trop les allumettes ${ }^{1}$ de Gaétan Soucy, cela ne soit pas le cas. Bien au contraire, nos deux protagonistes questionnent, voire rejettent le modèle éducatif reçu et tentent d'en créer un nouveau à partir de leurs propres principes et croyances. Ainsi, nous nous sommes demandé comment ils grandiront dans leur société respective où la privation de la mère, dans un premier temps, puis la disparition du père, dans un second temps, jouent un rôle majeur dans leur développement personnel. Mais aussi quel genre d'individu vont-ils devenir? Poursuivront-ils les lignes de conduite imposées par leur père, la tribu, la société québécoise ? Ou choisiront-ils un nouveau chemin, différent en tout et pour tout du modèle offert durant des années ? Car d'un côté, nous avons une histoire racontée par un narrateur extrahétérodiégétique se déroulant chez les Inuits dans les années 1940 ; de l'autre, une narratrice extra-homodiégétique dans un temps anhistorique, mais nous pouvons supposer que l'histoire se déroule dans les années 1980. Les deux auteurs québécois choisissent deux types de narration différente. En effet, Thériault opte pour une narration à la troisième personne, ce qui lui permet de représenter un point de vue extérieur sur la tribu autochtone. En d'autres termes, représenter le regard qu'un homme blanc peut porter sur les premières nations. Tandis que Soucy donne directement la parole à son personnage principal et central de l'histoire afin de créer un lien de proximité et d'intimité entre ce dernier et le lecteur.

$\mathrm{Au}$ cours de cette étude, nous avons donc choisi d'étudier les comportements des adolescents privés de leur père. Dans un premier temps, nous étudierons leurs réactions face à la disparation de la figure paternelle, puis nous nous intéresserons à leur évolution personnelle 
pour enfin observer leurs modes de survie mis en place dans la société. Nous verrons qu'il s'agit effectivement de « survie » puisque Agaguk décide de quitter la communauté inuit et de vivre sans la protection et la sécurité qu'elle peut offrir ; tout comme Alice qui devra affronter la vraie réalité de la société québécoise dont elle a été coupée pendant des années.

\section{Comment vivre sans le père ?}

Avant toute analyse, il est important de souligner la différence ethnographique et sociologique des deux œuvres à l'étude. Agaguk est un roman écrit par un auteur «Blanc» sur les Inuits dans les années 1940. Il semblerait que l'un des buts soit de témoigner de l'impact de la colonisation sur les tribus au Canada comme le souligne l'avertissement au début du roman: «L'action de ce roman se déroule chez les Esquimaux tels qu'ils étaient dans les années quarante. Que leur vie soit aujourd'hui modifiée par l'invasion du progrès dans l'Arctique est indéniable ». À travers cette histoire, l'auteur veut rendre justice aux Inuits en racontant une histoire qui leur serait la plus fidèle possible. $L P F$ est un roman écrit dans les années 1950 qui relate les évènements d'une fille et de son frère vivant dans un manoir, reculé du village et en totale autarcie. Il est intéressant d'analyser comment les deux personnages principaux évoluent dans le nouveau monde qu'ils se sont créé (Agaguk) ou qui a été imposé $(L P F)$, à des époques différentes et dans des contextes sociaux différents.

À la mort de leur père, les fils ${ }^{2}$ Soissons de $L P F$, endossent la lourde responsabilité de devoir « prendre l'univers en main » (1) et doivent apprendre à se débrouiller seuls. D'ores et déjà, nous remarquons qu'Alice crée de nouvelles expressions qui seraient au plus proche de sa perception de la réalité. En effet, l'expression que le lecteur pourrait s'attendre à lire, est «prendre sa vie en main » qui contient déjà une forte connotation de l'importance des choix à prendre au cours de sa vie. Ici, Alice permute la focalisation induite par «sa main » (qui est plus personnelle et individuelle) sur «l'univers » comme si elle assumait le rôle de maîtresse suprême du monde entier, mais à aucun moment, elle ne considère sa propre personne. Elle serait comme investie d'une mission dans laquelle sa vie serait mise au service des autres. Tandis qu'Agaguk, ayant métaphoriquement tué son père en le reniant lorsque ce dernier avait décidé d'épouser une Montagnaise, a décidé de quitter et d'abandonner sa tribu une fois la maturité atteinte : «quand il eut atteint l'âge et prouvé sa vaillance, Agaguk prit un fusil, une outre d'eau et un quartier de viande séchée, puis il partit à travers le pays [...] » (3). Dans les deux romans, la question du choix est soulevée ; pour l'un, cela s'est fait délibérément et en pleine conscience, Agaguk avait une volonté de rompre avec les traditions ; pour l'autre, la narratrice, Alice, n'avait pas choisi de devenir l'enfant le plus responsable des deux, cela lui a 
été imposé lorsque le père a décidé de se suicider, puisque c'est elle qui va devoir se rendre au village pour lui acheter un cercueil. Cette différence de choix remonte à l'éducation que chaque personnage a reçue. En effet, Alice et son frère vivent dans un manoir, loin du village où ils ont très peu interagi avec le monde extérieur : «il me faut parler de nos semblables, à mon frère et à moi, qui étaient environ au nombre de quatre » (34). En l'absence de la mère ou de figure maternelle, seul le père éduque ses enfants en leur imposant un code de conduite religieux régi par «les douze articles du code de la bonne maison» (14). Toutefois, ils ont aussi à disposition une bibliothèque d'où il semblerait que la jeune narratrice tienne ses histoires de preux chevaliers et de princesses. Leur éducation est donc très limitée comme en témoignent les nombreux néologismes, les comportements inappropriés et les réactions simplistes d'Alice lorsqu'elle se rend au village pour acheter un cercueil : «c'est très simple. Vous et votre bambin vous me donnez le cercueil, je vous donne mes sous, on met la dépouille dedans, et ensuite on creuse le trou qui va avec, en bordure de la pinède » (50-51). De plus, les deux enfants ne connaissent ni la valeur de l'argent - « il aurait dû nous apprendre la valeur de l'argent, fit mon frère » (24) - ni les codes sociaux à adopter devant les étrangers. Or, ils ne remettent jamais en question leur éducation, comme lorsqu'Alice décrit les relations sexuelles qu'elle et son frère entretiennent, ou encore lorsqu'elle parle de l'existence du Juste Châtiment (sa sœur jumelle, grande brûlée), enfermée dans la cave. Alice se contente de tenir à jour le journal quotidien, sans y apporter de jugements personnels. Il est évident que malgré tous les livres présents dans la bibliothèque, aucun d'entre eux n'aborde le thème de l'inceste ou tout simplement offre une éducation appropriée sur la sexualité. Nous avons affaire à une famille dysfonctionnelle où, rappelons-nous, le père exigeait de ses enfants un rituel de torture à respecter. Leur vie est très limitée et archaïque, par exemple Alice se sert d'un cheval comme moyen de transport et décrit la moto de l'inspecteur des mines comme : «un bourdon géant » (144). N'ayant eu pour point de repère que l'éducation donnée par leur père, il est clair que ces adolescents sont mentalement limités et ne savent pas comment se comporter en société. Tout porte à croire que l'absence de la mère a joué un rôle indéniable dans leur développement personnel.

De l'autre côté, Agaguk incarne la figure du jeune homme prêt à quitter le nid et à s'assumer pleinement. Il décide de rompre avec les traditions autochtones et de s'enfuir avec Iriook, l'adolescente qu'il a choisie pour femme: «il ne partageait avec personne cette contrée qu'il habitait, avec personne, sauf sa femme qu'il avait choisie et à qui il était attaché » (293). Ainsi, il ne suit plus les règles de la tribu, à savoir vivre en communauté, partager la nourriture, partager les femmes aussi, mais décide de mener une vie cachée, 
reculée, loin de sa famille et de ses congénères. Il adopte donc un style de vie proche de la culture européenne. Pour lui, la lignée a été rompue depuis que son père a pris pour femme une Montagnaise, c'est-à-dire une étrangère. Agaguk illustre ce que Martine-Emmanuelle Lapointe et Laurent Demanze appellent le processus de « dé-familiarisation » (8) : «L'héritier [...] n'est pas toujours condamné à porter le poids du passé. Si plusieurs auteurs confèrent une valeur éthique à la sauvegarde du legs, d'autres choisissent, au contraire, d'en jouer, non pas étrangers aux tragédies de leur époque, mais bien résolus à les dépasser » (7). En effet, Agaguk rejette son passé et part construire une nouvelle vie avec de nouvelles règles et codes de vie qui le mèneront à autoriser sa femme à parler pour lui à la suite de sa défiguration (246) et à accepter de garder leur deuxième enfant : «il ne voulait plus obéir aux traditions. La fille vivrait, parce qu'Iriook le voulait ainsi » (327). Agaguk se distingue clairement des traditions ancestrales et ouvre la porte à plus d'équité dans son couple. Ainsi, dans les deux romans, les personnages principaux sont capables de vivre sans leur père, soit en reproduisant et en imitant ce qui a été appris $(L P F)$, soit en s'éloignant et en refusant le schéma traditionnel de la tribu (Agaguk).

Dans son article «Fonctions freudiennes du père », Paul-Laurent Assoun rappelle le travail psychanalytique de Freud sur les relations mère-enfant et père-enfant tiré de l'étude de l'œuvre de Sophocle, Edipe Roi. En ce qui concerne le processus d'identification du fils envers le père, nous apprenons que: «Le fils en revanche a à suivre la voie de l'identification. Il ne s'agit pas seulement de s'identifier au père, mais de s'identifier au point de vue du père en quelque sorte [...] » (35). Bien qu'Alice soit une fille, pendant toute la première partie du roman, elle se considère comme un garçon. Ainsi, il est intéressant de remarquer que le processus d'identification au père s'est fortement opéré chez elle et non chez son frère. Elle n'a jamais remis en question son identité, voire son individualisation puisque le père les appelait soit «fils », soit «l'autre». Malgré son jeune âge, c'est elle qui remplacera la figure paternelle et assoira son autorité face à un frère perturbé. Rappelons-le, c'est elle qui va découvrir le cadavre de son père, elle aussi qui va aller au village chercher un cercueil et c'est elle qui, peu avant que son père ne se suicide, s'occupait du Juste Châtiment (sa sœur jumelle). La découverte du cadavre sera l'élément déclencheur et libérateur du récit que nous allons lire.

Toutefois, Agaguk va inconsciemment et métaphoriquement recréer l'un des mythes fondateurs de la culture européenne : le complexe d'CEdipe. En ne reconnaissant plus son père comme figure d'autorité, et surtout en ne le respectant plus à cause de son union avec une Montagnaise, Agaguk va tuer toute image et toute représentation de son père. Ce dernier sera 
relégué au rang de pur étranger extérieur à la vie d'Agaguk. Son père représente une tradition avec laquelle il ne s'identifie plus. N'ayant plus de modèle patriarcal à suivre, il part donc construire son propre modèle, avec ses propres règles, lois et valeurs.

\section{Leur évolution}

Dans les romans à l'étude, les deux personnages principaux s'émancipent et quittent le cocon familial pour aller affronter la réalité inquiétante et parfois dangereuse du monde extérieur, qu'elle soit celle de la toundra pour Agaguk ou du village pour Alice. Cette confrontation les mène vers une transformation et une acceptation de soi. Tel est le cas pour Agaguk qui, après avoir affronté le loup blanc subit non seulement une métamorphose physique, mais aussi psychologique. Au début, même s'il rompt avec les traditions en allant vivre ailleurs, il continue à perpétuer les rites de la tribu tels que revendre les peaux des animaux aux trafiquants anglais en échange de fusils. Ce qui le transformera à jamais, sera son duel avec le loup blanc, esprit vengeur du meurtre qu'il a commis contre Brown. Sur un autre plan symbolique, ce combat représente également la bataille entre les Inuits et les Blancs venus conquérir le territoire canadien. Réel tournant dans le récit, le combat le délivrera de ses obligations et devoirs patriarcaux pour permettre une permutation des rôles au sein du couple, c'est-à-dire vers plus d'équité entre l'homme et la femme. De plus, lorsqu'Ooanak demandera à Agaguk de devenir chef, ce dernier refusera catégoriquement de reprendre la place de son père. En effet, il ne s'agit pas simplement de remplacer le chef, il s'agit avant tout de remplacer le père de la tribu toute entière, car qu'est-ce qu'un chef si ce n'est la représentation symbolique du père ? En acceptant, Agaguk deviendrait un père pour tous ses congénères et se devrait d'incarner la voix du peuple, leurs lois, règles, valeurs et coutumes. En refusant ce rôle, il s'inscrit dans « la double injonction » imposée par le père que Sabine Prokhoris extrait du chapitre «le moi et le ça » des Essais de Psychanalyse de Freud (274), à savoir : "'Tu dois être ainsi' (comme le père) et 'Tu n'as pas le droit d'être ainsi' (comme le père)» (183). Agaguk a depuis longtemps compris le rôle de futur chef qui l'attendait si son père venait à mourir. Nous pouvons penser que l'une des raisons pour lesquelles il a quitté la tribu est ce refus d'être comme son père. Cela illustre également l'interdiction imposée par le père du non-droit que le fils a de ressembler à son père. Agaguk a très bien compris qu'il n'avait pas le droit d'être comme le père (celui de la tribu), mais il n'a surtout pas envie de l'être, car pour lui, il abandonnerait ce qu'il a de plus précieux à ses yeux, à savoir sa liberté, une liberté de choix de vie et de pensée. Revenir à la tribu serait un emprisonnement dans une essence qu'il a rejetée dès sa maturité. Comme l'explique Demanze, «les figures parentales 
ne fournissent ni modèles ni repères à l'aune desquels se constituer ». Pour Agaguk, son père est mort au moment où ce dernier a décidé de prendre une nouvelle femme. La stabilité que le couple parental pouvait lui offrir, a été rompue et par conséquent, ne lui permettait plus de s'identifier à ce nouveau modèle. Ainsi Agaguk évolue seul et contre la référence paternelle donnée. Il se construit individuellement et selon ses propres codes ${ }^{3}$.

Dans $L P F$, Alice va également se construire toute seule à la mort de son père. Toute sa vie elle a été considérée comme un garçon par son père et son frère (même après avoir perdu ses « couilles » et commencé à avoir ses règles). Son genre n'a jamais été remis en question par qui que ce soit puisque la famille vivait en autarcie. Les seuls contacts qu'ils ont eus avec le monde extérieur, s'élèvent au nombre de quatre, comme nous l'avons vu plus haut. Et les seules références provenaient soit du père, soit des livres de la bibliothèque. Ce père défroqué pousse le narcissisme à son plus haut point en ne considérant ces enfants qu'en tant que garçons où leur vie est réglementée et organisée par des rituels de torture. Cependant, en créant ce système, le père conditionne ses fils à ce qu'ils deviennent des «minis lui », car c'est en répétant ce que l'on voit ou l'on entend que les enfants se forgent. Le père les conditionne et les prépare à devenir de futurs pères (dans le sens d'exactes copies du père qu'il est déjà). Par conséquent, le père d'Alice peut espérer de continuer de vivre à travers ses enfants. C'est seulement à la suite de son interrogatoire avec l'inspecteur qu'Alice va commencer à « appliquer aux mots le genre des putes et les accorder en conséquence » (85). Jusqu'à ce passage-ci, le lecteur ignorait l'identité sexuelle de la narratrice puisque tous les mots étaient masculinisés : «j'étais le secrétarien ce jour-là » (13). Ce magnifique mot-valise combinant la fonction de secrétaire à la notion du rien. Ce travail (être secrétarien) incarne à merveille le rôle qu'Alice et son frère tenaient pendant toutes ses années : écrire dans le vide, écrire sur du rien, mais écrire tout de même. La mort du père la délivrera de son enfermement physique (elle ne se souvient pas de s'être rendue au village auparavant) et mental (elle prend conscience de sa physionomie et sait qu'elle va donner naissance à un enfant). L'écriture, bien que d'être un devoir imposé par le père (164), contribuera à sa libération identitaire puisque vers la fin du roman, elle confie avoir « toujours un peu su [qu'elle] était une pute» (167). De plus, grâce à l'écriture, elle arrivera à débloquer sa mémoire et à se souvenir des expériences de son passé, notamment de l'identité du Juste Châtiment, sa sœur jumelle. D’avoir entrepris la mission de se rendre au village et par la suite de poursuivre son écriture coûte que coûte, Alice a pu libérer sa mémoire du joug paternel. Tenir un journal était ce qui la maintenait en vie, malgré l'absence de vrais mots ou de phrases réelles, pour elle, les lignes qu'elle traçait, représentaient sa pensée. Dans son article « Tombeaux de l'enfance. Pour une prosopopée de 
la mémoire chez Émile Nelligan, Réjean Ducharme et Gaétan Soucy », Jean-François Hamel explique l'importance pour un enfant d'entreprendre un récit libéré de toute pression parentale : «l'énonciation assumée par l'enfant lui-même, et non par un adulte qui se poserait avec autorité comme son avenir, présente l'enfant ici et là en train d'écrire et, plus étrangement encore, de se souvenir » (98). Alice est désormais capable de se souvenir des évènements qui se sont réellement passés et donc de faire la distinction entre fiction et réalité. Ainsi il semblerait que pour survivre dans le nouveau monde que les deux personnages Agaguk et Alice - se sont imposé, il faille remettre en question l'autorité paternelle.

\section{La survie}

Après avoir vécu sans figure paternelle, se pose donc la question de la survie. Comment Agaguk et Alice arrivent-ils à survivre sans leur (re)père ? Dans Agaguk, le narrateur extra-hétérodiégétique - qui permet au lecteur d'avoir un recul sur le récit -, raconte en détail la vie d'Agaguk et d'Iriook. Le lecteur suit pas à pas leur évolution, mais aussi leur passage de jeunes adolescents à parents avec la naissance de Tayaout en premier, puis des jumeaux à la fin du livre. Cette transition vers le statut parental est très importante, car il permet de se rendre compte des comportements adoptés par les jeunes parents envers leur progéniture. Comme tout parent attentionné et aimant, Agaguk et Iriook considéreront la naissance de Tayaout comme un événement merveilleux : « on le chante » (93). Selon la tradition esquimaude, il est important d'avoir des garçons plutôt que des filles, car forts, robustes, et chasseurs, ils assureront la survie de la tribu (316). Ainsi, c'est une réussite pour le jeune couple puisqu'il était primordial d'avoir un garçon afin de subvenir aux besoins familiaux. Dès lors, Agaguk commence à s'imaginer ce qu'il apprendra à son fils : «je lui enseignerai comment construire l'igloo [...], comment tailler un couteau à neige à même les os d'un phoque, ou avec une côte de caribou... » (101), autrement dit, comment survivre dans la toundra. Inconsciemment, il commence à endosser le rôle de père et naïvement il croit qu'il va se différencier de son propre père en éduquant Tayaout à l'art de la survie. Agaguk ne se rend pas compte qu'en faisant de la sorte, il reproduit le schéma parental de base : enseigner à ses enfants ce que nous avons appris. Bien entendu, puisqu'Agaguk a décidé de partir de la tribu, nous pouvons supposer qu'il ne va enseigner que ce qu'il considère «bon » pour son fils et qu'il va laisser le négatif de côté. Or, il ne nous sera jamais révélé si plus tard Tayaout aura accepté cette éducation, car il n'est pas improbable que ce dernier suive les pas de son père (Agaguk) et refuse cette vie solitaire pour revenir aux traditions inculquées par la tribu. Dans « La fuite et le retour aux sources dans Agaguk d'Yves Thériault », Renald Bérubé rappelle 
qu'Agaguk a choisi un vieux prénom pour son fils : «Agaguk remonte dans la lignée des vrais Inuit pour trouver quelqu'un qui soit digne d'être continué ; les qualités du Tayaout ancien sont toutes nobles et sont à l'opposé de celles qui font les héros au pays de Ramook » (79). Agaguk remonte dans le passé afin de recréer une société qui correspond à ses attentes lorsqu'il avait décidé de quitter la tribu. La totale négation et oblitération de son père prouve qu'il est prêt à proposer un nouveau modèle à ses enfants, à l'image de la modernité.

Il semblerait que dans un premier temps, Alice se libère du joug paternel qui est double chez elle puisque son père était aussi prêtre. Les deux fils Soissons vivent selon les préceptes de la Bible lus très littéralement et malheureusement pas toujours compris. Cette délivrance va lui permettre d'accéder à une prise de conscience générale sur sa condition. En effet, elle arrive à se poser des questions sur le rôle paternel et sur son éducation : «j'ai crainte que nous n'ayons rien fait que continuer à lui obéir, sans le vouloir [...]. Peut-être n'avons-nous jamais cessé d'être ses poupées de cendre » (164). Elle est désormais capable de produire sa propre réflexion et de se rendre compte des faiblesses ou des erreurs de son père. C'est ce qu'évoque Dominique Viart dans « Le silence des pères au principe du 'récit de filiation' » lorsqu'il explique comment, dans les récits de filiation, le respect filial impose que l'on « ne montre pas les faiblesses, les erreurs, ni les errements de ses parents » (106). Jusqu'à présent, Alice n'avait fait son devoir de «secrétarien », à savoir retranscrire mot pour mot (du moins le croit-elle) les évènements quotidiens de la maison, sans jamais y apporter son jugement personnel. À présent, elle ose soulever le problème, mais n'ira pas jusqu'à rejeter la faute sur son père. En effet, bien qu'elle décide d'élever sa fille dans une société entièrement matriarcale et dépourvue d'horion (178), cette dernière se verra attribuer le lourd nom d'Ariane « en mémoire du châtiment... » (179). Par cet acte, elle condamne sa fille à de futurs problèmes identitaires, car elle incarnera la voix de la mort, la voix du silence et de l'absence, c'est-à-dire sa tante. En cela, elle illustrera une vieille figure rhétorique, la prosopopée, qui consiste «à mettre en scène et à faire parler des absents, des morts, des choses, des êtres inanimés, à donner la parole à ce qui par nature en est dépourvu (Hamel 97). L'onomastique des enfants sert à se remémorer le passé ; qu'il soit glorieux et source de renouveau chez Agaguk ou espérance naïve et souvenir d'un passé douloureux à ne pas reproduire pour $L P F$.

Dans «La fonction paternelle, question de théorie ou : des lois à la Loi », Françoise Hurstel développe les trois fonctions de la «paternité »: « la fonction, le rôle et la personne du père » (243). La fonction serait « [...] de l'ordre du langage et de la parole, elle introduit du sens pour un sujet [...] »; le rôle est «l'ensemble des conduites concrètes attendues, soit par la société, soit par la famille ${ }^{4} »$ et enfin la personne qui est «appelée père doit être [...] 
distinguée de la fonction et du rôle. Un homme est désigné pour porter ce nom de père, pour assurer ce rôle dans la famille [...]»(243-244). Le père serait donc cette figure qui devra assumer un rôle prédéterminé par le langage, la société et enfin la famille. Il sera la figure centrale autour de laquelle tous les autres membres de la famille se réfèreront. Ainsi, être père - ou parent - n'est pas une tâche aisée : il faut incarner une personne autre que soi-même, il faut laisser de côté l'individu pour laisser place au parent chargé d'éduquer son enfant. C'est ce que nos deux nouveaux parents, Agaguk et Alice, vont apprendre à faire. Ils vont endosser, voire embrasser la lourde responsabilité d'être parent et par conséquent incarner ces trois fonctions.

L'une des premières responsabilités qu'un parent a, est l'appellation de son enfant. Le nom que l'on choisit, détermine qui l'on sera. Ainsi, il n'est pas anodin qu'Agaguk décide de nommer son fils d'après un nom ancestralement significatif. Pour lui, ce sera afin de retrouver des valeurs traditionnelles perdues. Agaguk impose à son fils un héritage que ce dernier aura la lourde tâche de perpétrer et d'honorer. La démarche d'Alice est légèrement différente de celle d'Agaguk, car nommer son enfant signifie lui attribuer une identité propre, qu'elle soit individuelle ou sexuelle. Forte de sa prise de conscience et de sa nouvelle maturité, Alice comprend qu'il est important pour sa fille de grandir dans un environnement où cette dernière sera reconnue en tant qu'individu unique.

\section{Conclusion}

Le récit de filiation propose au lecteur une relecture du passé filial afin d'en comprendre l'héritage identitaire laissé par les parents. La mort du père dans Agaguk et LPF provoque la remise en question des traditions et du système éducatif aboutissant à une émancipation et une acceptation de soi. Les deux jeunes adolescents choisiront de reproduire certains schémas tels que la biparentalité pour Agaguk et la monoparentalité pour Alice, restes d'un mimétisme éducationnel, tandis que d'autres seront rejetés. La survie des personnages principaux passerait donc par la reproduction. Ce sont les nouvelles générations qui permettent à nos personnages de survivre dans leurs nouvelles conditions puisqu'ils doivent (ou devront) à leur tour s'occuper et éduquer leurs enfants afin qu'ils développent les capacités cognitives suffisantes pour vivre dans le monde. En d'autres termes, nous avons affaire à un éternellement recommencement de la vie avec ses qualités et ses défauts. 


\section{Bibliographie}

Assoun, Paul-Laurent. «Fonctions freudiennes du père ». Le père : métaphore paternelle et fonctions du père : l'interdit, la filiation, la transmission. Dir. Marc Augé. Paris : Denoël, 1989. 25-51.

Bérubé, Renald. «La fuite et le retour aux sources dans Agaguk d'Yves Thériault ». Voix et images du pays 1.1 (1970). 71-82.

Demanze, Laurent. «Récits de filiation». Fabula (août 2008). http://www.fabula.org/atelier.php?R\%26eacute\%3Bcits_de_filiation

Hamel, Jean-François. « Tombeaux de l'enfance. Pour une prosopopée de la mémoire chez Émile Nelligan, Réjean Ducharme et Gaétan Soucy ». Globe : revue internationale d'études québécoises 4.1 (2001). 93-118.

Hurstel, Françoise. «La fonction paternelle, question de théorie ou : des lois à la Loi ». Le père: métaphore paternelle et fonctions du père: l'interdit, la filiation, la transmission. Dir. Marc Augé. Paris : Denoël, 1989. 235-262.

Lapointe, Martine-Emmanuelle et Laurent Demanze. «Présentation : figures de l'héritier dans le roman contemporain ». Études françaises 45.3 (2009). 5-9.

Prokhoris, Sabine. «Entre dire et ne pas dire, interdire : Ambigüités de la fonction du père ». Le père: métaphore paternelle et fonctions du père: l'interdit, la filiation, la transmission. Dir. Marc Augé. Paris : Denoël, 1989. 183-195.

Soucy, Gaétan. La petite fille qui aimait trop les allumettes. Montréal : Boréal, 2000.

Thériault, Yves. Agaguk. Montréal : Typo, 1993.

Viart, Dominique. «Le silence des pères au principe du 'récit de filiation' ». Études françaises 45.3 (2009). 95-112.

\section{Notes}

${ }^{1}$ Désormais, nous indiquerons le roman sous l'acronyme $L P F$.

${ }^{2}$ Il convient de noter que pendant toute la première partie du roman, la narratrice s'identifie comme un garçon, tout du moins, croit-elle en être un :« Frère m'appelle frère, et père nous appelait fils quand il nous commandait tout la veille encore $\gg(81)$.

${ }^{3} \mathrm{~N}$ 'oublions pas que nous ne naissons pas parents, nous le devenons.

${ }^{4}$ Définition reprise des sociologues (Segalen, M. Sociologie de la famille. Paris : Armand Colin, 1981) 